\title{
AMCoR
}

Asahikawa Medical College Repository http://amcor. asahikawa-med. ac. jp/

Respirology (2007) 12(3):458-461.

Radiological findings of alveolar hydatid disease of the lung caused by Echinococcus multilocularis.

Ohsaki Y, Sasaki T, Shibukawa K, Takahashi T, Osanai S. 


\section{Radiological Findings of Alveolar Hydatid Disease of the Lung Caused by}

\section{Echinococcus multilocularis}

Yoshinobu Ohsaki ${ }^{1}$, Takaaki Sasaki ${ }^{1}$, Kiyoko Shibukawa1, Toru Takahashi ${ }^{1}$, Shinobu

Osanai 1

${ }^{1}$ Division of Respiratory Diseases, Department of Medicine, Asahikawa Medical College.

2-1-1-1 Midorigaoka Higashi, Asahikawa 078-8510, Japan.

Phone +81/166/68-2442

Facsimile +81/166/68-2449

Corresponding author

Yoshinobu Ohsaki, MD, PhD

e-mail yohsaki@asahikawa-med.ac.jp

Key words: Echinococcosis, Alveolar hydatid disease, Echinococcus multilocularis, chest radiography, computed tomography.

Running title: Radiological findings of alveolar hydatid disease. 


\section{Abstract}

Alveolar hydatid disease is a highly malignant form of echinococcosis caused by the larvae of the cestode Echinococcus multilocularis. Alveolar hydatid disease always affects primarily to the livers, unlike cystic hydatid disease caused by E. granulosus. Occasionally, there are metastases to the lungs and brain from the primary lesions. There have been few reports on the radiological features of alveolar hydatid disease caused by E. multilocularis. In the present report, we describe the radiological features of alveolar hydatid disease of the lung caused by E. multilocularis. In the cases presented here, multiple nodules were noticed on the chest radiogram. These nodular shadows varied in size and shape. On computed tomographs, the majority of nodular shadows were lobulated and well circumscribed. These nodules were triangular, spherical, oval or linear in shape. We considered that multiple lobulated lesions located between two segments of the lung and showing various shapes were characteristic of pulmonary alveolar hydatid disease caused by E. multilocularis. 


\section{Introduction}

Alveolar hydatid disease is a highly malignant form of echinococcosis caused by the larvae of the cestode Echinococcus multilocularis. E. multilocularis enters the human body via contaminated drinking water and infects the liver. Progressive larval spread within the liver in a cancer-like fashion leads to death from hepatic insufficiency. Alveolar hydatid disease always affects primarily the liver, unlike cystic hydatid disease caused by $E$. granulosus. Occasionally, there are metastases in the lungs and brain. Echinococcosis caused by E. multilocularis is an endemic disease in Hokkaido, Japan.

Four Echinococcus strains - E. granulosus, E. multilocularis, E. vogeli and E. oligarthus - have been reported to be pathogenic to humans. These four species of Echninococcus are morphologically distinct in their adult and metacestode stages, geographic distribution and host specificity (1). E. granulosus is widely distributed throughout the world because of its very low intermediate host specificity and its high prevalence in sheep and cattle herding locals. E. multilocularis is restricted to the northern hemisphere, with endemic areas extending from the European alpine countries, the former Soviet Union, China, Alaska, South-Central Canada, North of the United States, and Hokkaido where the fox appears to serve as the definitive host and certain wild rodents as intermediate hosts. The cycle involves these canids and rodents including voles, muskrats, meadow voles, and house 
mice. In settlement areas, domestic dogs and cats may feed on these rodents, contaminate water and vegetation, and in this way, human beings become accidental intermediate hosts. E. vogeli and E. oligarthus are restricted in their distribution to South and Central America. The host assemblages include the bush-dog and paca and a wild feline cycle involving the puma/jaguar/Geoffroys cat and agouti/paca (1).

Majority of E. multilocularis infections arise solely in the liver. Pulmonary involvement is seen in 5 to $10 \%$ of the cases with hepatic echinococcosis caused by $E$. multilocularis. Wilson et al. found only three cases of pulmonary involvement among 33 cases of alveolar hydatid disease (2). The radiological features of pulmonary hydatid disease caused by E. granulosus are well documented; multiple cysts are seen in $20 \%$ to $30 \%$ of the patients (3-6). Whereas there have been few reports on the radiological features of alveolar hydatid disease caused by E. multilocularis.

In the present report, we describe the radiological features of alveolar hydatid disease of the lung. 


\section{Cases of $\boldsymbol{E}$. multilocularis}

Case 1 is a 59-year-old woman. She underwent surgical resection of a large soft tissue tumor in the anterior chest wall ten years previously. The tumor was diagnosed as a granuloma caused by E. multilocularis based on pathological findings. Besides hepatic lesions, multiple pulmonary lesions were found on the chest radiogram and the patient was referred to the Division of Respiratory Diseases. Her chest lesions have been monitored for more than ten years.

Case 2 is a 51-year-old woman who has been treated for hepatic echinococcosis caused by E. multilocularis for 12 years already. Hepatic lesions were detected after surgical resection of the right iliac bone affected by E. multilocularis. Besides the hepatic lesions, multiple pulmonary lesions were detected on the chest radiogram and she was referred to the Division of Respiratory Diseases. She has been followed up for more than seven years. These pulmonary lesions have gradually increased in size during follow-up. Serological examination was performed in Asahikawa Medical College and the results were positive for E. multilocularis in both cases (7).

\section{Findings on the Chest Radiogram}

Multiple nodules were detected on the chest radiogram of case 1 . There were at least three nodules in the right lung field, and one in the left lung field. In case 2, more than 
ten nodules including a $2.5 \mathrm{~cm}$ mass were noticed in both lung fields on the chest radiograph. These nodular shadows varied in size and shape. Both patients were treated using albendazole. Pulmonary lesions were decreased in size during the therapy and increased in size by termination of the therapy. Figure 1 shows chest radiogram of case 2 .

\section{Findings on the Chest Computed Tomography}

The diameter of these pulmonary nodules varied from several $\mathrm{mm}$ to $2.5 \mathrm{~cm}$. These lesions located between two segments of the lung, and pulmonary veins which distribute between these segments were involved. The majority of nodular shadows were lobulated and well circumscribed. Nodules were either triangular, spherical, oval or linear in shape. These findings seemed to be characteristic of pulmonary alveolar hydatid caused by $E$. multilocularis (figure 2). 


\section{Discussion}

Alveolar hydatid disease is a consequence of metastasis of the larvae of the cestode $E$. multilocularis from the primary lesion. The parasitic cycle involves fox and rodents, which contaminate water and vegetation, and it occasionally involves domestic dogs. In Japan, systemic parasitic diseases are usually seen as imported cases from endemic regions (8) except for the disease caused by E. multilocularis.

In the present observations, we found that the radiological appearance of the pulmonary alveolar hydatid of E. multilocularis varied in size and shape. Each pulmonary lobe is divided into individual segments by inter segmental connective tissue and segmental veins. Segmental veins can be differentiated from segmental arteries by HRCT because these arteries locate at the center of the segment in parallel with the segmental bronchus. The characteristic radiological findings of pulmonary alveolar hydatid disease in the present cases included inter-segmental distribution involving these segmental veins. It has been documented that rarely, tumor emboli can be seen in segmental or larger arteries, and seldom, centrilobular nodules and branching liner opacities (tree-in-bud pattern) can be seen in metastatic pulmonary neoplasms (9). Probably, alveolar hydatid disease spreads in a manner similar to that of metastatic neoplasms. We consider this pathomechanism of alveolar hydatid disease as one of the reasons for the distribution of the lesions in this location. Nodules were 
lobulated in shape, increased in density and well circumscribed. Also, absence of marked retraction of adjacent organs such as pleura, bronchus and pulmonary vessels seemed to be characteristic of pulmonary alveolar hydatid disease caused by E. multilocularis. Cavity formation was not seen in our cases.

Most pulmonary metastatic tumors have smooth margins, probably because they grow relatively uniformly in all directions from their original focus (9); although nodules with spicules or poorly defined margins can also be seen. Primary pulmonary adenocarcinoma is typically observed as a spherical or lobulated lesion, well circumscribed or showing margins retraction of the pleura into the tumor and a distinctive puckered appearance indicating retraction of adjacent organs (10). Usually this characteristic appearance is seen only in primary adenocarcinoma; metastases from the primary lesion lack these characteristics. These differences in radiological features differentiate pulmonary alveolar hydatid disease from metastatic tumors and primary adenocarcinomas of the lung. However, the radiological differentiation of alveolar hydatid disease of the lung from other multiple pulmonary parasitic diseases may be difficult.

Most commonly, pulmonary involvement is evident in the right lower lobe of the lung because of direct spread by the hematogenous route. Our cases lack such tendency of distribution, however, more lesions were seen in the right lung than in the left one. Treugut 
et al. reported that radiological findings of alveolar hydatid disease of the lung can be classified into two types (11). Type 1 lesions denote hematogenous spread and result in multiple small, irregular, peripheral lesions. Type 2 manifestations are caused by local extension of the hepatic mass resulting in focal pulmonary involvement, which may be difficult to distinguish from a pulmonary abscess. According to this classification, pulmonary nodules in the present cases were type 1 lesions.

Compare to the development of unilocular hydatid cyst caused by E. granulosus, the alveolar hydatid caused by E. multilocularis develops very differently. The germinal layer of the metacestode proliferates both exogenously and endogenously. This form of development and infiltration of the host tissues is facilitated by the relative absence of the pericyst and a very thin laminated layer. The metacestode mass thus consists of vesicular tissue composed of 5- to 15-mm-size vesicles that progressively invade the host tissue. Cells from the germinal layer can detach and metastasize to distant organs (1). We speculate that these distinctive features in the pathogenesis of the alveolar hydatid caused by E. multilocularis are responsible for the radiological features of the disease in the lungs.

The symptoms of these pulmonary lesions are usually overshadowed by the manifestations of the primary infectious burden of the liver (2). None of our patients complained of respiratory symptoms. Alveolar echinococcosis by E. multilocularis caused 
up to $100 \%$ lethality in untreated patients before the 1970s. Benzimidazole derivatives, mebendazole and albendazole, treatment have shown some promise in the treatment. However, a very high oral dose for three months to more than 17 years is needed to control the disease because of their variable absorptions.

Acknowledgements

Authors thank Professor Akira Ito of Department of Parasitology, Asahikawa Medical College for his helpful suggestions. 


\section{References}

1. Bhatia G. Echinococcus. Semin Respir Infect 1997;12:171-186

2. Wilson JF, Raush RL. Alveolar hydatid disease. A review of clinical features of 33 indigenous cases of Echinococcus multilocularis infection in Alaskan Eskimos. Am J Trop Med Hyg 1980;29:1340-1355

3. Saksouk FA, Fahl MH, Rizk GK. Computed tomography of pulmonary hydatid disease. $J$ Comput Assist Tomo 1986;10:226-232.

4. Amir-Jahed AK, Fardin R, Farzad A, Bakshandeh K. Clinical echinococcosis. Ann Surgery 1975;182: 541-546

5. Sadrieh M, Dutz W, Navabpoor MS. Review of 150 cases of hydatid cyst of the lung. Chest 1967;52: 662-666

6. Beggs I. The radiology of hydatid disease. AJR 1985;145:639-648

7. Fujimoto Y, Ito A, Ishikawa Y, et al. Usefulness of recombinant Em18-ELISA to evaluate efficacy of treatment in patients with alveolar echinococcosis. J Gastroenterol 2005;40:426-431

8. Ohsaki Y, Matsumoto A, Miyamoto K, et al. Neurocysticercosis without detectable specific antibody. Internal Med 1999;38:67-70

9. Muller NL, Fraser RS, Lee KS, Johkoh T. Miscellaneous neoplasms. In: Diseases of the lung. Philadelphia, PA: Lippincott Williams \& Wilkins, 2003:110-135.

10. Muller NL, Fraser RS, Lee KS, Johkoh T. Pulmonary carcinoma. In: Diseases of the lung. Philadelphia, PA: Lippincott Williams \& Wilkins, 2003: 76-96

11. Treugut H, Schulze K, Hubener KH, Andrasch R. Pulmonary involvement by Echinococcus alveolaris. Radiology 1980;137:37-41 


\section{Figure legends}

Figure 1. Chest radiogram of case 2. More than ten nodules including a $2.5 \mathrm{~cm}$ mass were noticed in both lung fields

Figure 2. Forty nodules were found in case 1 and case 2. Upper: 17 nodules were detected by high-resolution computed tomography scanning. Lower: 23 nodules were detected by conventional computed tomography scanning. Each figure is $3 \times 3 \mathrm{~cm}$ in size. 
Figure 1. Radiological Findings of Alveolar Hydatid Disease by Y. Ohsaki et al.

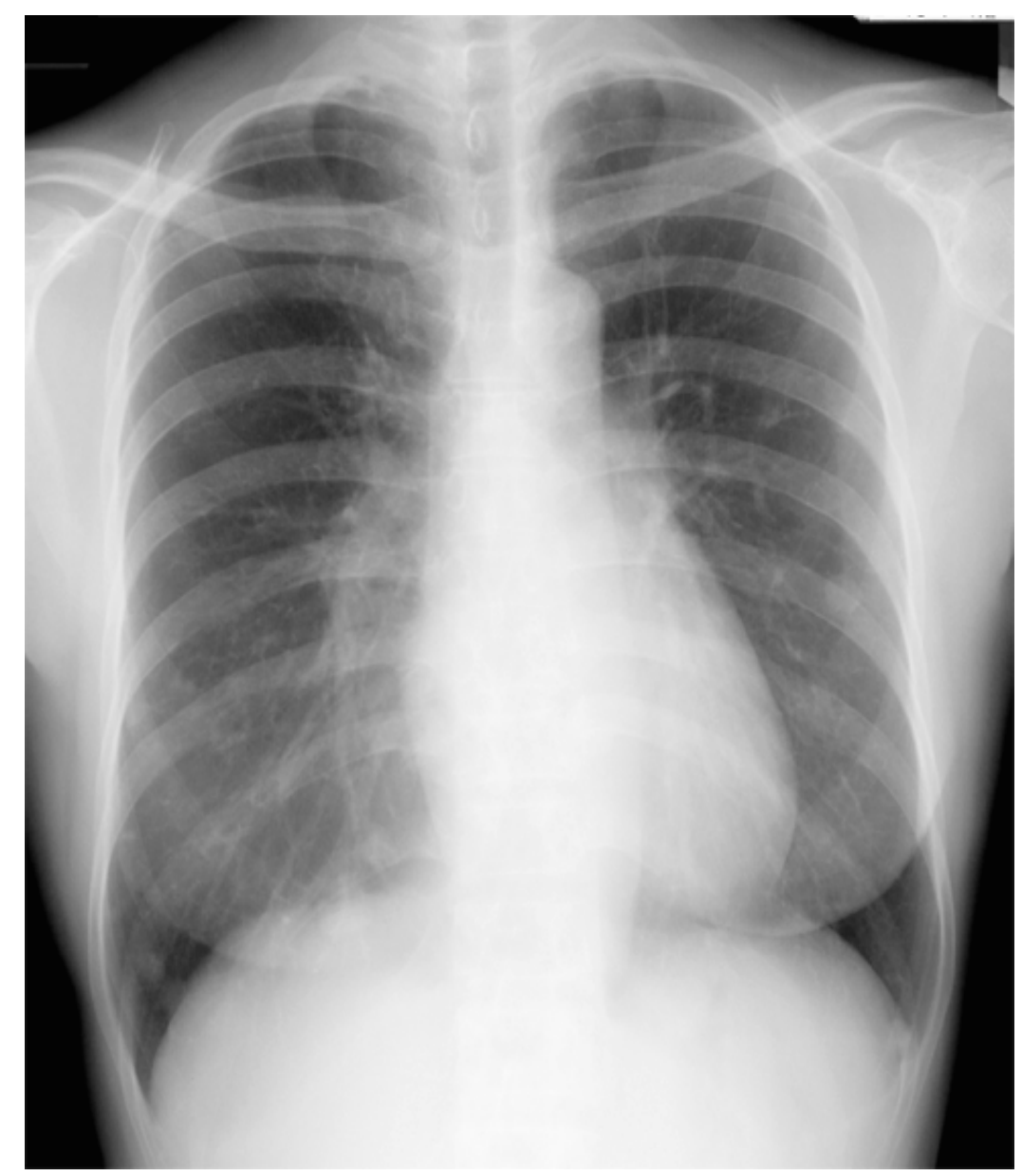


Figure 2. Radiological Findings of Alveolar Hydatid Disease by Y. Ohsaki et al.
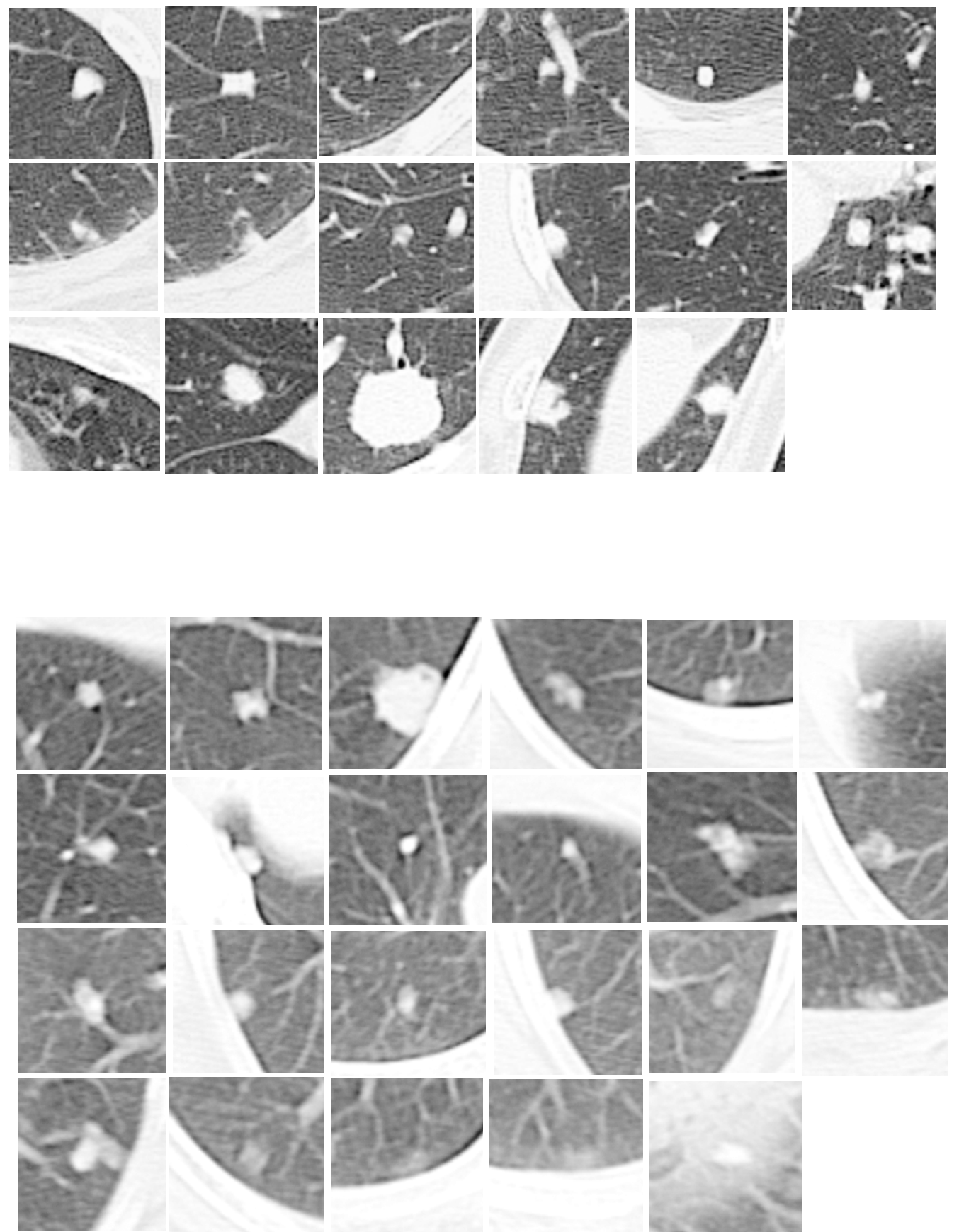